\title{
Guidelines for aute medical management of severe traumatic brain injury in infants and children
}

\section{Praveen Khilnani}

The guidelines for the acute medical management of severe traumatic brain injury in infants, children, and adolescents were published on June 62003 as special supplements to Pediatric critical care medicine, Critical Care Medicine and the Journal of Trauma, ensuring a multidisciplinary audience of more than 30,000 speciality physicians. According to brain injury association of America, more than 1 million children in the US sustain brain injuries each year, of whom 250000 are admitted to hospitals, 7000 die, and 30,000 are permanently disabled. Indian statistics though not available should be comparable at the least. Following is a summary of available evidence in the literature.this information could be used to apply to care of head injures infants and children in the Indian scenario.

\section{Evidence highlights}

1. Pediatric patients with traumatic brain injury should be treated in a pediatric trauma center or, failing that, a tertiary care hospital with pediatric trauma care capability.

2. Hypoxia must be treated appropriately; however, there is no evidence to support endotracheal intubation versus bag-mask ventilation during transfer to the hospital.

3. Prophylactic treatment with mannitol or mild hyperventilation is usually unnecessary but should be used in patients with evidence of cerebral herniation or worsening neurological function.

4. Intracranial pressure monitoring is indicated for children with a Glasgow coma score of less than 8,but it may also be employed for children in whom serial neurological examination is not feasible.

\section{From:}

Department of Paediatrics, Indraprastha Apollo Hospital, Sarita Vihar,

New Delhi - 110044, India.

Correspondence:

Dr. Praveen Khilnani

B-42, Panchsheel Enclave, New Delhi - 110017, India

E-mail: praveenk@nde.vsnl.net.in
5. Treatment for increased intracranial pressure should be initiated when the pressure rises more than 20-25 $\mathrm{mm} \mathrm{Hg}$

6. The sensitivity of ventricular catheters, external guage transducers, or catheter tip pressure transducers in monitoring intracranial pressure appears equal. Subarachnoid, subdural,epidural, and externally placed monitors are less accurate.

7. Cerebral perfusion pressure should be maintained at more than $40 \mathrm{~mm} \mathrm{Hg}$. Further research is needed to determine an optimal cerebral perfusion pressure range.

8. The routine use of sedation and neuromuscular blockade in severe pediatric traumatic brain injury is not supported by scientific evidence.

Coughing and bucking on the tube and suctioning maneuvers leading to increased intracranial pressure may necessitate their use, in practice.

9. Cerebrospinal fluid drainage via ventriculostomy is a first line option for refr actory elevated intracranial pressure; lumbar drainage may be added in patients with open cisterns on imaging and without major mass lesions or midline shift.

10.Mannitol or hypertonic saline are both acceptable agents for lowering intracranial pressure.

11. Hyperventilation should only be used as a second line method to reduce refractory intracranial pressure. In this setting, the paCO2 should be kept at 25-30 mmhy.

12. High dose barbiturates may also be employed in the treatment of refractory increased intracranial pressure. Patients receiving this therapy require extremely close monitoring for hypotension

13. Hyperthermia has been postulated to increase secondary mechanisms of brain injury in adults. Therefore, hyper thermia should be avoided in younger patients. Hypothermia on the other hand, may be ben- 
eficial, and when intracranial hypertension is refractory, the authors recommend that it be considered, despite lack of evidence.

14.Decompression craniectomy may also be considered to improve refractory intracranial hypertension. Surgical interventions may be more successful in patients with reversible brain insults.

15. There is no evidence to recommend steroid therapy in children with traumatic brain injury.

16. Although research had not directly addressed outcomes in pediatric patients with traumatic brain injury, nutritional support should be strongly considered with a goal of $130 \%-160 \%$ of resting metabolic expenditure.

17.Prophylactic antiepileptic medications are not recommended.

\section{Conclusion}

1. Prompt recognition and transfer of pediatric victims of traumatic brain injury is paramount to good outcomes

2. Intracranial pressure should be monitored and treated if $>20 \mathrm{~mm}$ hg in children with severe brain injury

3. Mannitol, hypertonic saline, ventricular drainage, hyperventilation, barbiturates and hypothermia should be considered in refractory intracranial hypertension

4. Steroids and prophylactic antiepileptics have no role in traumatic brain injury in children.

\section{Reference}

Guidelines for the acute medical management of severe traumatic brain injury in infants,children, and adolescents:Pediatr Crit Care Med 2003:4:3:S1-72 